Check for updates

1 Division of Biosciences, University College London, London, UK

2 Department of Health Services Research and Policy, London School of Hygiene and Tropical Medicine, London, UK

3 Institute of Population Health, University of Liverpool, Liverpool, UK

4 European Observatory on Health Systems and Policies, London, UK

Correspondence to: A Crozier alexander.crozier.20@ucl.ac.uk Cite this as: BMJ 2021;372:n208 http://dx.doi.org/10.1136/bmj.n208 Published: 03 February 2021

\title{
Put to the test: use of rapid testing technologies for covid-19
}

\author{
Alex Crozier and colleagues look at how new technologies can be most appropriately used to \\ support different testing strategies and examine the benefits and risks
}

Alex Crozier, ${ }^{1}$ Selina Rajan, ${ }^{2}$ lain Buchan, ${ }^{3}$ Martin McKee ${ }^{2,4}$

Governments have invested enormous resources in scaling up testing capacity in their responses to covid-19. Real-time reverse transcription polymerase chain reaction (PCR) was the first, and still the most widely used, test. However, several days can elapse between requesting a test and getting and acting on a result, leaving a window in which infection may spread. A further problem is that people may transmit infection before recognising symptoms ${ }^{1}$-a key driver of spread. In addition, some people who remain asymptomatic have a similar viral load to those who develop symptoms and may also contribute to spread, although the extent of this is unclear. ${ }^{1-3}$ Given the importance of presymptomatic or paucisymptomatic transmission, ${ }^{1}$ measures that shorten the time between testing and results are essential for minimising onward transmission. It is difficult to achieve this with large scale PCR testing.

Rapid antigen lateral flow tests offer an alternative. They provide a rapid result but are less able to detect infections. ${ }^{4}$ Governments are purchasing them in large quantities, with some seeing a single test as a way to free an individual from quarantine obligations, a view not supported by the World Health Organization. ${ }^{4}$ However, repeated lateral flow testing may have value in combination with other measures. In Liverpool, UK, for example, authorities are examining test-to-protect, test-to-release (from quarantine), and test-to-enable (safer return to restricted activities) regimens, alongside outbreak response and public open access to lateral flow testing. ${ }^{5}$ Such real world evaluations are needed to understand how these models work in different populations and settings, how they influence behaviour, and the contribution of lateral flow tests to overall strategies, where they have the potential to interrupt transmission while reducing the mounting harms from restrictions.

\section{Interpreting test results}

Meaningful interpretation of any test requires knowledge of its sensitivity (proportion of infected people who test positive), specificity (proportion of non-infected people who test negative), and pre-test probability that an individual is infected, reflecting population prevalence and the individual's circumstances. $^{6}$

Although controls within assays minimise errors, technical problems during sample collection, processing, or reporting can give false results. Lateral flow tests produce very few false positive results, and in a low prevalence setting these can be detected by confirmatory PCR testing. ${ }^{4} 7$ False negative results are more concerning. Besides technical errors, they can arise in people tested during the 5-7 day incubation period before the viral antigen shed in the nose and throat is sufficient to be detected, usually 1-2 days before symptom onset. ${ }^{128}$ Taking swabs requires skill, and swabs taken by untrained individuals are more likely to give false negative results. ${ }^{910}$ False negatives might create a false sense of security, paradoxically increasing transmission risk. ${ }^{11}$

Conversely, PCR testing is overly sensitive, detecting viral shedding long after the infectious period (about 9 days), with people continuing to test positive for a mean of 17 days. $^{2}$ Although such positive results are technically correct, these people are not infectious and should not be quarantined. Moreover, any test is just a snapshot of the moment the sample was taken.

A shared understanding of the utility and uncertainties of these tests is key to using them well (see bmj.com for a full analysis). ${ }^{12}$ Effective communication of what results mean is paramount.

\section{Novel tests}

Several novel techniques, such as loop mediated isothermal amplification, next generation sequencing (LamPORE), point-of-care PCR, and lateral flow tests are in different stages of development, validation, approval, and implementation (see appendix table 1 on bmj.com). Each has advantages and limitations, so the choice depends on the intended use. Lateral flow tests (table 1) aim to detect only infectious cases, can be scaled up quickly for decentralised testing, are relatively cheap, do not require laboratories, and provide results rapidly. As such, they are most appropriate for widespread community testing intended to reduce transmission or time spent in isolation, and to enable economic and social activities to restart. Lateral flow tests are less sensitive than nucleic acid amplification tests such as PCR, generating more false negative results if used as a test of infection rather than infectiousness, and they are particularly susceptible to sampling quality. ${ }^{413}$ 
Table 1| Advantages and limitations of lateral flow tests compared with PCR tests*

\begin{tabular}{|c|c|}
\hline Advantages & Limitations \\
\hline Rapid time to results (10-30 minutes) & Some infectious individuals will have negative results \\
\hline Does not need laboratory analysis and so can facilitate frequent decentralised testing at scale & $\begin{array}{l}\text { End-to-end single test performance falls when used by untrained staff or public-less so when } \\
\text { repeated }\end{array}$ \\
\hline $\begin{array}{l}\text { Good detector of the most infectious cases and less likely to detect post-infectious people with } \\
\text { residual shedding }\end{array}$ & $\begin{array}{l}\text { Infectious window is early and short lived, narrowing the window to find cases before they } \\
\text { transmit infection }\end{array}$ \\
\hline $\begin{array}{l}\text { Effective contact tracing depends on speed, and modelling suggests testing frequency and } \\
\text { speed of reporting more important than sensitivity alone for surveillance and controlling } \\
\text { transmission }\end{array}$ & $\begin{array}{l}\text { Current lateral flow test does not quantify the level of virus material detected to reflect a level } \\
\text { of infectiousness }\end{array}$ \\
\hline
\end{tabular}

* See appendix for performance results.

The window for using lateral flow tests to detect infectious cases is narrow. ${ }^{214}$ They are most suitable when testing is frequent, as we explain below, and the goal is detection of cases with high viral shedding immediately before and after symptom onset. ${ }^{24}$ Despite their limitations, their ability to provide near instantaneous results avoids the delays associated with PCR and facilitates timely isolation of the most infectious cases and their close contacts, ${ }^{415}$ who may otherwise transmit infection while waiting for a PCR result.

Although lateral flow tests have a higher risk of false negative results, in theory the rapid increase in viral shedding after the incubation period leaves only a short period when there will be a substantial difference between the point when you get a first positive result on a highly sensitive test (PCR) compared with a lower sensitivity test (lateral flow). ${ }^{16}{ }^{17}$ Importantly, modelling suggests more frequent testing with lower sensitivity tests can achieve the same probability of detecting a case as less frequent testing with higher sensitivity tests (fig 1). ${ }^{18} 19$ Under laboratory conditions, the limit of the detection capacity of lateral flow tests largely aligns with the quantities of viral shedding (quantified as viral load) and ability to culture virus typically observed at the end of the first week of symptoms, ${ }^{1420}$ after which most patients cease being infectious. ${ }^{2}$

\section{- Viral load \\ — Low analytic sensitivity (lateral flow test) - - - High analytic sensitivity (PCR) \\ O Negative test \\ o Positive test}

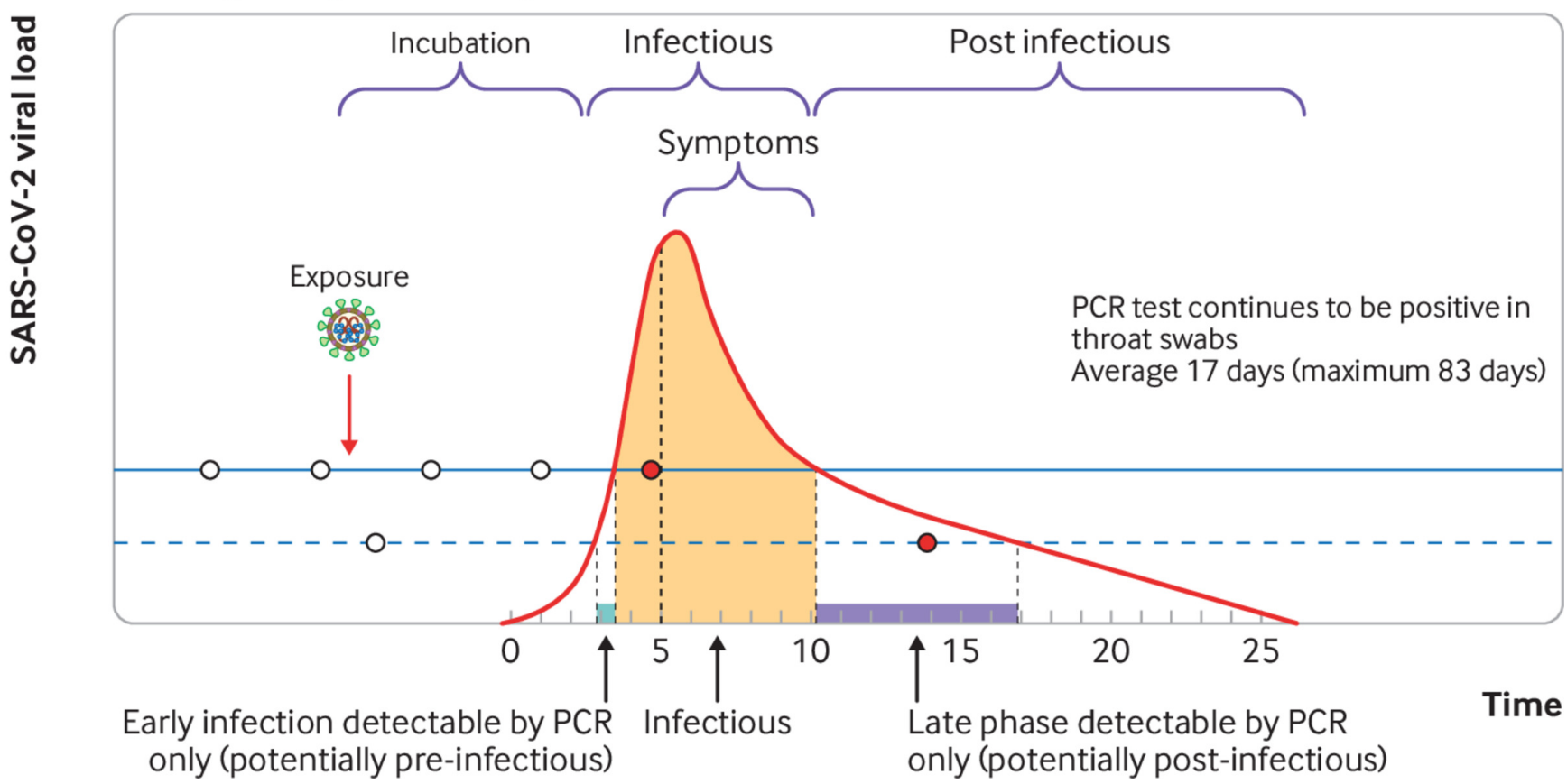

Fig 1 | High frequency testing with low analytic sensitivity versus low frequency testing with high analytic sensitivity. A person's infection trajectory (blue line) is shown in the context of two surveillance regimens (circles) with different analytic sensitivity. Higher frequency testing is more likely to test in the infectious window. Therefore, although both testing regimens detect the infection (orange circles), the high frequency lateral flow test is more likely to detect it during the transmission window (shading), despite its lower analytic sensitivity. The figure is not an accurate representation of exactly when a positive test is likely to signify that a case is infectious. Adapted with permission from Mina et $\mathrm{al}^{16}$ with data from Cevik et $\mathrm{al}^{12}$

As detected viral antigen and cell culture infectivity are proxies and not direct indicators of infectiousness, caveats remain, but the point when lateral flow test results change from negative to positive, and vice versa, mostly coincides with the beginning and end of infectiousness of most symptomatic cases, ${ }^{14}$ and potentially also in asymptomatic cases. Furthermore, analysis of national PCR and 
contact tracing data showed that cases with high viral load are the most infectious (that is, infect more of their contacts) and that, under laboratory conditions, the best performing lateral flow tests detect $91 \%$ of cases that lead to onward transmission (based on modelled estimates with implicit uncertainty and dynamics). ${ }^{21}$ Thus, despite their lower sensitivity, lateral flow tests may be a useful indicator of current infectivity and less likely than PCR to detect post-infectious shedders.

Test sensitivity is operator dependent, ${ }^{10}$ and self-swabbing in real world conditions is likely to miss more infections than swabbing in controlled conditions. In the Liverpool community testing pilot, samples were obtained by self-swabbing under supervision of soldiers, and lateral flow tests missed $60 \%$ of PCR positive cases. ${ }^{25}$ However, over half of these people with positive PCR results were likely to be post-infectious shedders, consistent with the low and falling prevalence in Liverpool at the time (see appendix). ${ }^{5}$ Although further work is required to determine how detected viral load relates to infectiousness, data suggest lateral flow tests missed less than a third of people likely to be infectious. ${ }^{521}$ Use and interpretation of lateral flow devices can improve with more robust operating procedures, additional training, and possibly AI augmented reading. ${ }^{510}$ Antigen tests and population groups are also heterogenous, so test accuracy must be understood for different groups (eg, asymptomatic or paucisymptomatic, and by age and background prevalence) before large scale use.

\section{Testing strategies}

Countries have adopted different testing strategies, many using lateral flow tests (fig 2). Their benefits and risks are summarised in the appendix on bmj.com. Each should be evaluated, recognising that testing can only be one part of a comprehensive pandemic response. When deciding which test to adopt, and how to implement it, system-wide practicalities must be considered, especially accessibility and acceptability of sampling, turnaround times, and re-test intervals.

Focused symptomatic testing

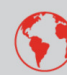

Symptomatic testing

Confirm case diagnosis and rapidly trace contacts, testing only those with symptoms. Japan has focused on cluster identification

Focused asymptomatic testing

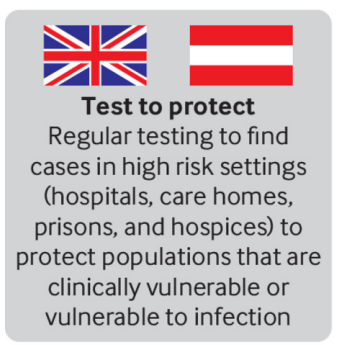

vulnerable to infection

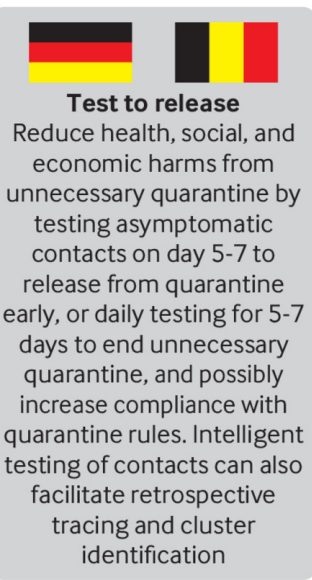

Reduce health, social, and economic harms from necessary quarantine by testing asymptomatic contacts on day 5-7 to rease from quarantine days to end unnecessary quarantine, and possibly increase compliance with quarantine rules. Intelligent facilitate retrospective and cluster

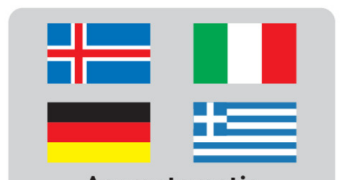

Asymptomatic international arrivals Reduce quarantine time and socioeconomic impact (and possibly increase compliance) by testing international arrivals on arrival, or at day 5-7

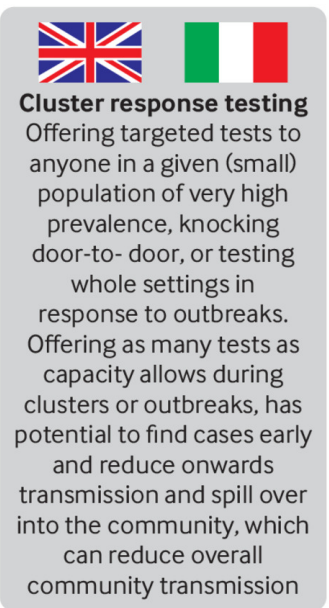

Test to enable Enable return to otherwise restricted activities of health, social, or economic importance. Make "covidfree" bubbles by screening out positive cases through regular testing of groups susceptible to transmission at a place of work or education, to gain entry to an event, or care home visiting (where evidence supports)

Mass testing

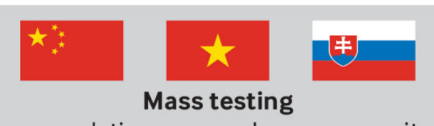

Mass testing

Mass community case finding in high prevalence populations may reduce community transmission to more manageable levels, or in low prevalence populations mass repeat testing stop community transmission in a given population through early identification of cases

Fig 2 | Principal testing strategies and examples of countries deploying them. Summary of the benefits and risks of each testing strategy and the biochemical limitations and other challenges to large scale asymptomatic testing are described in the appendix on bmj.com

\section{Mass testing}

The effectiveness, feasibility, opportunity costs, and ethics of large scale asymptomatic testing are fiercely debated. Some have likened this to cancer screening programmes, but the pandemic context is quite different. Cancer screening aims to benefit the individual whereas testing for the presence of highly transmissible respiratory infections is to protect others by breaking transmission chains.

Mass (repeat) testing for SARS-CoV-2 using PCR in China, Vietnam, and Iceland and lateral flow tests in Slovakia has identified cases that would have gone undetected. However, mass testing poses tremendous logistical challenges, requiring considerable resources and careful planning. In the UK, non-focused mass repeat testing is unlikely to be feasible or cost effective.

\section{SMART}

Liverpool, UK, has developed a focused approach to community testing with lateral flow tests called SMART (systematic meaningful asymptomatic repeated testing). Open access testing of the public is supported by communications and outreach targeting specific groups that are vulnerable to covid-19 or its control measures. SMART comprises a dual strategy of focused reduction in 
transmission alongside outbreak response and specific test-to-protect, test-to-enable, and test-to-release schemes designed to protect key services, reconnect societies, and recover the economy. The benefits and risks of the scheme are monitored through public health, healthcare, and administrative data and continuous qualitative information gathering. This information is used to adapt the evolving programme in weekly reviews, co-created with community groups. For example, when the UK introduced a national lockdown at the start of 2021, testing in Liverpool focused on workplaces to enable continuity of essential services and to protect against transmission in high mixing environments such as supermarkets. Positive lateral flow results are confirmed with PCR and viral genetic sequencing.

Although SMART has considerable potential to find asymptomatic or paucisymptomatic cases early and reduce onwards transmission, large scale use is resource intensive and requires effective local engagement. Participation in mass testing in China, Vietnam, and Slovakia was compulsory, with quarantine enforced and consequences for non-adherence. Given the importance of presymptomatic and paucisymptomatic transmission, SMART must find more cases in the incubation period to improve on symptomatic testing, which in theory it should, while reducing the risks from false negative results. Up to 21 January 2021, 359606 lateral flow tests had been done on 205836 Liverpool residents, identifying 4421 people who may not have otherwise known they were likely to be infectious (CIPHA dashboard data, available from authors on request). These people were then able to self-isolate, breaking chains of onward transmission.

Although the behavioural responses to large scale asymptomatic testing in the community are not fully understood, particularly the potential increase in hazardous behaviours following a negative result, Office for National Statistics survey data in Liverpool showed most $(62 \%)$ said a negative result would be unlikely to cause them to change their behaviour. ${ }^{5}$ However, some said they were more likely to visit friends ( $9 \%$ ) or go to work $(7 \%)$, emphasising the need to communicate the importance of maintaining covid safe behaviours. The considerable advantages of identifying infectious cases early to break transmission chains must be balanced against any potential false reassurance given to people with false negative results and the risks that confirmed cases do not isolate. This balance, and programme cost effectiveness, are the keys to realising the net benefits of SMART.

Although $95 \%$ of positive cases in the Liverpool pilot self-isolated and informed relevant contacts immediately, not being able to afford the costs of self-isolation was a substantial barrier to uptake, 5 highlighting the need for a holistic public health approach, including effective communication and comprehensive support to self-isolate. Tests alone are not the answer: recent experience of repeat lateral flow testing in German care homes highlights the many logistical, economical, and behavioural challenges of SMART. ${ }^{22}$

Logistical factors are important, such as arrangements for booking and queuing for tests, so the choice of test policy will often be limited by cost, available workforce, and capacity of the community to access booking systems and testing sites. The biological, behavioural, ethical, and system implications of complex public health interventions such as SMART must be evaluated, with findings used to develop rigorous standard operating procedures and protocols that optimise strategies (fig 3). Communicating this evidence clearly is essential to achieve the? public and professional trust needed if testing is to succeed. 


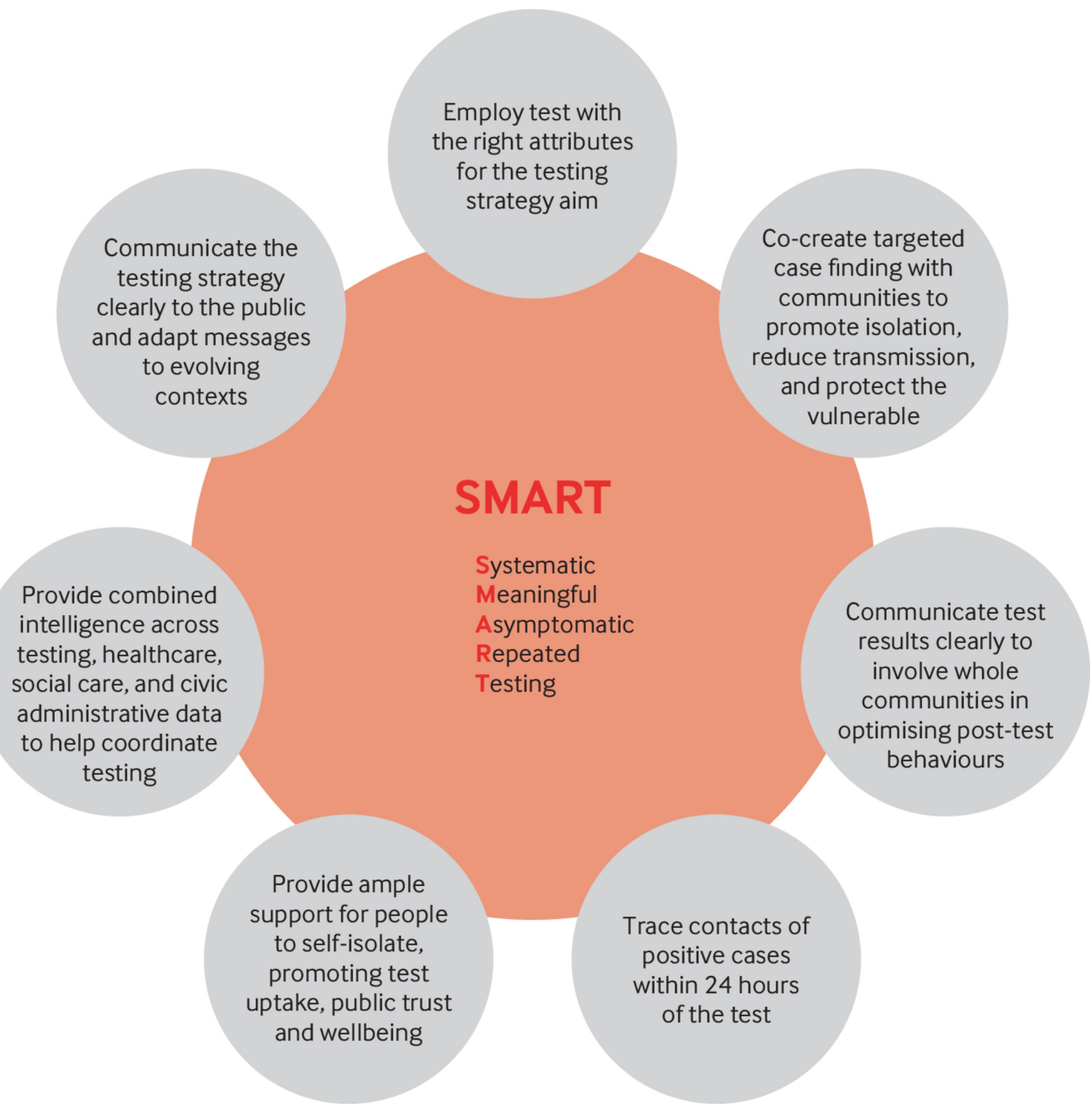

Fig 3 | Keys to successful SMART (systematic meaningful asymptomatic repeated testing) programme

\section{Improving cluster identification and outbreak response}

It takes 4-5 days for someone infected with SARS-CoV-2 to infect another person so contact tracing must identify and reach contacts as soon as possible. ${ }^{23}$ As many people don't request a test until at least 24-48 hours after developing symptoms and PCR can take more than 48 hours to return results (median 38 hours with $14 \%$ taking over 72 hours most recently in England ${ }^{24}$ ), substantial onwards transmission can occur before contacts are reached and clusters of cases can quickly develop into large outbreaks. Modelling suggests rapid and frequent testing can help to reduce transmission. ${ }^{19} 25$ In high risk settings for outbreaks (workplaces, care homes, schools, universities, prisons, and hospitals), repeated and frequent lateral flow tests cut these delays, providing real time results for cases and close exposure contacts, which can identify clusters ${ }^{26}$ quickly and limit spread. To mitigate the increased risks of false negative results, people with symptoms could have lateral flow and PCR tests in parallel. Robust communication that a negative lateral flow result does not mean "not infectious" is essential, and symptomatic people must continue to isolate.

Cases could also be identified earlier if the clinical case definition were broadened to include symptoms such as disturbance (rather than loss) of taste and smell, headaches, and myalgia, which often present earlier than the classic symptoms. Although existing testing capacity might struggle, a broader definition would be more consistent with WHO criteria ${ }^{27}$ and should be evaluated. Outbreak response and community testing can also be improved using mobile or pop-up PCR or LamPORE laboratories, which can provide relatively high throughput sensitive testing with a turnaround time 
of 4-24 hours. Cluster identification can also be improved by using genomic sequencing technology, which can inform contact tracing and potentially reduce transmission. ${ }^{28}$

\section{Test to protect}

If implemented carefully, repeated testing in high infection risk settings can protect people who are either clinically vulnerable or vulnerable to infection (and transmission). ${ }^{29}$ Although the high sensitivity of the PCR test favours its use in vulnerable settings, it can take days from requesting a swab to getting the result. Frequent, rapid decentralised lateral flow testing may prove more effective.

The recent policy of bi-weekly testing of NHS staff with lateral flow tests recognises that frequent testing can compensate for reduced sensitivity. Specific testing strategies may also be focused on protecting groups most susceptible to infection and transmission, such as key workers, enabling continuity of essential services and possibly reducing overall transmission. Again, communication must be clear that a negative result does not necessarily mean a person is non-infectious. Real world implementation and practicalities must also be considered and optimised. Crucially, test-to-protect policies will have limited effect unless workers are supported to self-isolate. ${ }^{30}$ Weekly point-of-care PCR testing is also being evaluated in some UK care homes, for both staff and visitors, but more frequent lateral flow testing is also being evaluated and may be more (cost) effective.

\section{Test to release}

Test-to-release models use repeated tests to reduce unnecessary quarantine of non-infectious people, focusing isolation decisions on presumed infectivity rather than on infection. Various modelling suggests that testing exposed contacts and international arrivals can shorten the duration of isolation, which is likely to increase compliance. ${ }^{31}{ }^{32}$ PCR testing has been used as a way to shorten unnecessary quarantine of non-infectious contacts, while daily lateral flow testing is being piloted as a way to end unnecessary quarantine of non-infectious contacts. However, PCR has a median false negative rate of $38 \%$ five days after exposure and $20 \%$ on day 8 (although some assays showed near $100 \%$ detection at day 7 ). 33 Although one study showed PCR testing on day 7 reduced quarantine time and resulted in no post-quarantine transmission, ${ }^{34}$ an evaluation of test-to-release of household contacts showed that $19 \%$ experienced symptoms or received a positive PCR test result after testing negative seven days after the index case developed symptoms, ${ }^{35}$ suggesting this is strategy is not without risk. Any test-to-release policy must account for the incubation period, ${ }^{32}$ mitigate the risks of premature return or hazardous behaviours (see bmj.com), and be shown to be cost effective. Ultimately, no test can replace comprehensive support, both practical and financial, 3036 as a means of tackling low rates of self-isolation, particularly in disadvantaged communities.

\section{Test to enable}

Test-to-enable policies seek to lift the current restrictions on social contact that are causing wider public health and economic harms in a way that minimises risk. For example, specific test-to-enable strategies may be able to reduce the harms of social isolation by enabling care home visiting, or to support workplaces in fragile local economies to operate (with risk mitigation). Focused regular testing is more logical than single tests for entry, which are unlikely to confer population wide benefits. ${ }^{11}$ Context is key: disadvantaged areas with greater mounting harms from measures to control covid-19 could get most benefit from locally sensitive responses. Again, however, practicalities and false negative results are concerns, and we must await quality pilot data before any large scale rollout.

\section{Conclusion}

Rapid tests provide new opportunities to find and isolate cases and contacts early in the infection. However, implementing such tests in local health systems is complex. Continued formative evaluation is needed if such testing is to simultaneously reduce transmission and alleviate the mounting harms from control measures. Pilots of the SMART approach provide evidence of large scale, targeted use of lateral flow tests. Successful approaches to control covid-19 must facilitate earlier and better targeted isolation of the most infectious people and their close contacts while, where the evidence supports, releasing non-infectious contacts sooner from unnecessary quarantine and returning to a more open society and economy. This requires testing to be integrated into a comprehensive programme, co-created with local leaders and communities, including effective contact tracing, appropriate support and credible incentives for those isolating, and strategic testing as part of ongoing surveillance of vaccine effectiveness. A holistic public health approach, joined up across towns, cities, and regions, is key to sustainable recovery from the covid-19 pandemic.

\section{Key messages}

- Strategies for use of covid-19 tests vary widely between countries

- Rapid tests provide opportunities for early detection and isolation but must be integrated into wider strategies to control transmission

- Assessment of the benefits and risks of different testing strategies suggests how novel tests can be used in public health policies to improve covid-19 resilience and recovery

Contributors and sources: The authors have broad experience and direct involvement in covid-19 responses. AC has expertise developing and troubleshooting diagnostic assays and improved covid-19 testing programmes for sports organisations. SR has supported the Public Health England regional response, including managing outbreaks in care homes and educational institutions and contributed extensively to the covid-19 Health Systems Response Monitor produced by the European Observatory on Health Systems and Policies in partnership with WHO. Martin McKee is a member of Independent SAGE and has published extensively on the pandemic. IB is leading evaluation of the Liverpool SMART pilot and NIHR senior investigator.

Competing interests: We have read and understood BMJ policy on declaration of interests and have no interests to declare.

Provenance and peer review: Not commissioned; externally peer reviewed.

Cevik M, Kuppalli K, Kindrachuk J, Peiris M. Virology, transmission, and pathogenesis of SARS-CoV-2. BMJ2020;371:m3862. doi: 10.1136/bmj.m3862 pmid: 33097561

2 Cevik M, Tate M, Lloyd O, Maraolo AE, Schafers J, Ho A. SARS-CoV-2, SARS-CoV-1 and MERS-CoV viral load dynamics, duration of viral shedding and infectiousness: a living systematic review and meta-analysis.SSRN2020. [Preprint.] doi: 10.2139/ssrn.3677918

3 Lee S, Kim T, Lee E, etal. Clinical course and molecular viral shedding among asymptomatic and symptomatic patients with SARS-CoV-2 infection in a community treatment center in the Republic of Korea. JAMA Intern Med 2020;180:1447-52.

doi: 10.1001/jamainternmed.2020.3862 pmid: 32780793

$4 \quad$ World Health Organization. Antigen-detection in the diagnosis of SARS-CoV-2 infection using rapid immunoassays. Interim guidance, 11 Sep 2020. https://apps.who.int/iris/handle/10665/334253

5 Liverpool covid-19 community testing pilot-interim evaluation report. 2020. https://www.liverpool.ac.uk/media/livacuk/coronavirus/Liverpool,Community, Testing,Pilot,Interim,Evaluation.pdf Watson J, Whiting PF, Brush JE. Interpreting a covid-19 test result. BMJ2020;369:m1808. doi: 10.1136/bmj.m1808 pmid: 32398230

Centers for Disease Control and Prevention. Interim guidance for rapid antigen testing for SARS-CoV-2. 2020. https://www.cdc.gov/coronavirus/2019-ncov/lab/resources/antigen-testsguidelines.html

8 Lauer SA, Grantz KH, Bi Q, etal. The incubation period of coronavirus disease 2019 (COVID-19) from publicly reported confirmed cases: Estimation and application. Ann Intern Med 2020;172:577-82. doi: 10.7326/M20-0504 pmid: 32150748

9 McCulloch DJ, Kim AE, Wilcox NC, etal. Comparison of unsupervised home self-collected midnasal swabs with clinician-collected nasopharyngeal swabs for detection of SARS-CoV-2 infection. JAMA Netw Open2020;3:e2016382. doi: 10.1001/jamanetworkopen.2020.16382 pmid: 32697321 
10 Porton Down PHE, University of Oxford. Preliminary report: rapid evaluation of lateral flow viral antigen detection devices (LFDs) for mass community testing. 2020.

https://www.ox.ac.uk/sites/files/oxford/media_wysiwyg/UKevaluation_PHEPortonDownUniversityofOxford_final.pdf.

11 Scientific Advisory Group for Emergencies. Multidisciplinary task and finish group on mass testing consensus statement for SAGE. 27 Aug 2020. https://www.gov.uk/government/publications/tfmsconsensus-statement-on-mass-testing-27-august-2020

12 McCartney M, Sullivan F, Heneghan C. Information and rational decision-making: explanations to patients and citizens about personal risk of COVID-19. Evidence-Based Med, 2020. [Epub ahead of print.], doi: 10.1136/bmjebm-2020-111541.

13 Dinnes J, Deeks J), Adriano A, etalCochrane COVID-19 Diagnostic Test Accuracy Group. Rapid, point-of-care antigen and molecular-based tests for diagnosis of SARS-CoV-2 infection. Cochrane Database Syst Rev 2020;8:CD013705. doi: 10.1002/14651858.CD013705. pmid: 32845525

14 Mühlemann B, Zuchowski M, Karen W, Lei J. Comparison of seven commercial SARS-CoV-2 rapid point-of-care antigen tests.medRxiv 2020.[Preprint] https://www.medrxiv.org/content/10.1101/2020.11.12.20230292v1.article-metrics

15 Bullard J, Dust K, Funk D, et al. Predicting infectious SARS-CoV-2 from diagnostic samples. Clin Infect Dis 2020;ciaa638.pmid: 32442256

16 Mina MJ, Parker R, Larremore DB. Rethinking covid-19 test sensitivity - a strategy for containment N Engl J Med 2020;383:e120. doi: 10.1056/NEJMp2025631 pmid: 32997903

17 Madewell Z), Yang Y, Jr IML, Halloran ME, Dean NE. Viral dynamics of SARS-CoV-2 infection and the predictive value of repeat testing.medRxiv 2020. [Preprint.] https://www.medrxiv.org/content/10.1101/2020.10.21.20217042v1

18 Lanièce Delaunay C, Saeed S, Nguyen QD. Evaluation of testing frequency and sampling for severe acute respiratory syndrome coronavirus 2 surveillance strategies in long-term care facilities. J Am Med Dir Assoc 2020;21:1574-1576.e2. doi: 10.1016/j.jamda.2020.08.022 pmid: 32994117

19 Larremore DB, Wilder B, Lester E, etal. Test sensitivity is secondary to frequency and turnaround time for COVID-19 surveillance.medRxiv 2020. [Preprint.] https:/www.medrxiv.org/content/10.1101/2020.06.22.20136309v3

20 Kohmer N, Toptan T, Pallas C, et al. The comparative clinical performance of four SARS-CoV-2 rapid antigen tests and their correlation to infectivity in vitro. J Clin Med2021 doi: 10.3390/jcm10020328

21 Lennard YWL, Rozmanowski S, Pang M, et al. An observational study of SARS-CoV-2 infectivity by viral load and demographic factors and the utility lateral flow devices to prevent transmission. 2021. http://modmedmicro.nsms.ox.ac.uk/wp-content/uploads/2021/01/infectivity_manuscript_20210119_merged.pdf

22 Ettelt S. Rapid COVID-19 testing in care homes in Germany: easier said than done. 2020 https://tccovid.org/2020/11/24/rapid-covid-19-testing-in-care-homes-in-germany-easier-saidthan-done/

23 Aghaali M, Kolifarhood G, Nikbakht R, Saadati HM, Hashemi Nazari SS. Estimation of the serial interval and basic reproduction number of COVID-19 in Qom, Iran, and three other countries: A data-driven analysis in the early phase of the outbreak. Transbound Emerg Dis2020;67:2860-8. doi: 10.1111/tbed.13656 pmid: 32473049

24 DHSC. Weekly statistics for NHS Test and Trace (England): 17 December to 23 December. 2020 https://www.gov.uk/government/publications/nhs-test-and-trace-england-statistics-17-decemberto-23-december/weekly-statistics-for-nhs-test-and-trace-england-17-december-to-23-december

25 Grassly NC, Pons-Salort M, Parker EPK, White PJ, Ferguson NMImperial College COVID-19 Response Team. Comparison of molecular testing strategies for COVID-19 control: a mathematical modelling study. Lancet Infect Dis 2020;20:1381-9. doi: 10.1016/S1473-3099(20)30630-7 pmid: 32822577

26 Endo A, Leclerc QJ, Knight GM, etalCentre for the Mathematical Modelling of Infectious Diseases COVID-19 Working Group. Implication of backward contact tracing in the presence of overdispersed transmission in COVID-19 outbreaks. Wellcome Open Res 2020;5:239. doi: 10.12688/wellcomeopenres.16344.1 pmid: 33154980

27 World Health Organization. Covid-19: case definitions. Aug 2020. https://www.who.int/publications/iitem/WHO-2019-nCoV-Surveillance_Case_Definition-2020.2.

28 Aggarwal DD, Fieldman T, Warne B. Genomic epidemiology of SARS-CoV-2 in the University of Cambridge identifies dynamics of transmission: an interim report. 2020. https://assets. publishing.service.gov.uk/government/uploads/system/uploads/attachment_data/file/950795/s0963genomic-epidemiology-sars-cov-2-university-of-cambridge.pdf

29 Houlihan C, Vora N, Byrne T, etal. SARS-CoV-2 virus and antibodies in front-line health care workers in an acute hospital in London: preliminary results from a longitudinal study.medRxiv 2020. [Preprint.] https://www.medrxiv.org/content/10.1101/2020.06.08.20120584v1

30 Crozier A, Mckee M, Rajan S. Fixing England's COVID-19 response: learning from international experience. J R Soc Med 2020;113:422-7. doi: 10.1177/0141076820965533 pmid: 33058751

31 Centers for Disease Control and Prevention. Options to reduce quarantine for contacts of persons with Sars-CoV-2 infection using symptom monitoring and diagnostic testing. 2020. https://www.cdc.gov/coronavirus/2019-ncov/more/scientific-brief-options-to-reduce-quarantine.html

32 Quilty BJ, Clifford S, Hellewell J, etal. Quarantine and testing strategies in contact tracing for SARS-CoV-2: a modelling study[Epub ahead of print.]. Lancet 2021;267:1-9.

33 Kucirka LM, Lauer SA, Laeyendecker O, Boon D, Lessler J. Variation in false-negative rate of reverse transcriptase polymerase chain reaction-based SARS-CoV-2 tests by time since exposure. Ann Intern Med 2020;173:262-7. doi: 10.7326/M20-1495 pmid: 32422057
34 Wells CR, Townsend JP, Pandey A, etal. Optimal COVID-19 quarantine and testing strategies.medRxiv 2020 [Preprint.] https://www.medrxiv.org/content/10.1101/2020.10.27.20211631v4

35 Rolfes MA, Grijalva CG, Zhu Y, etal. Implications of shortened quarantine among household contacts of index patients with confirmed SARS-CoV-2 infection-Tennessee and Wisconsin, April-September 2020. MMWR Morb Mortal Wkly Rep 2021;69:1633-7. doi: 10.15585/mmwr.mm695152a1 pmid: 33382676

36 Kerkhoff AD, Sachdev D, Mizany S, etal. Evaluation of a novel community-based covid-19 'test-to-care' model for low-income populations. PLoS One 2020;15:e0239400. doi: 10.1371/journal.pone.0239400 pmid: 33035216

This article is made freely available for use in accordance with BMJ's website terms and conditions for the duration of the covid-19 pandemic or until otherwise determined by BMJ. You may use, download and print the article for any lawful, non-commercial purpose (including text and data mining) provided that all copyright notices and trade marks are retained. 